\title{
El signo del surco profundo
}

Dras. Manuela Pérez $\mathrm{M}^{(1)}$, Karla Moënne $\mathrm{B}^{(2)}$, Patricia Bitar $\mathrm{H}^{(3)}$.

1. Fellow de Radiología Pediátrica, Clínica Las Condes-Universidad de Chile.

2. Radióloga Pediatra. Clínica Las Condes. Santiago - Chile.

3. Radióloga Adultos, Clínica Las Condes. Santiago - Chile.

The deep sulcus sign

Abstract. The "deep sulcus sign" is seen on anterioposterior (AP) chest radiographs obtained with the patient in the supine position, both in adults and children. It is an indication of a pneumothorax, since air collects within the nondependent portions of the pleural space abnormally deepening the costophrenic angle, which extends toward the hypochondrium.

Among other findings, this useful sign should be actively searched for when a pneumothorax is suspected in a supine patient.

Key words: Chest, Pneumothorax, Radiography.

Resumen. El "signo del surco profundo" se visualiza en radiografías (Rx) de tórax anteroposteriorestomadas en posición supina, tanto en niños como en adultos. Traduce la presencia de aire en el espacio pleural, que se dispone en la porción no dependiente y distiende anormalmente el receso costofrénico, proyectándose hacia el hipocondrioipsilateral. Constituye uno de los signos radiológicos más útiles para el diagnóstico de neumotórax en Rx obtenidas en decúbito, por lo que debe buscarse dirigidamente, en especial en las placas portátiles.

Palabras clave: Neumotórax, Tórax, Radiografía.

Pérez M, et al. El signo del surco profundo. Rev Chil Radiol 2012; 18(2): 85-87.

Correspondencia: Dra. Manuela Pérez M / maneperez@yahoo.com

Trabajo recebido el 20 de abril de 2012, aceptado para publicación el 8 de junio de 2012.

\section{Aspecto imaginológico}

El "signo del surco profundo" se visualiza en Rx de tórax tomadas en posición supina, en niños y adultos (Figura 1). Se observa como una asimetría de los ángulos costofrénicoslaterales,originada en mayor profundidad y radiolucidez de uno de ellos; el senopuede incluso extenderse hasta el hipocondrio y adoptar una morfología triangular o crescéntica ${ }^{(1)}$ (Figura 2).

\section{Reseña histórica}

Si bien ya había sido mencionado previamente, la primera publicación que describe el signo del surco profundo en el contexto de una serie de pacientes aparece en 1980 cuando Gordon, et al presentaron una serie de 60 pacientes con diagnóstico de neumotórax (adultos y niños), observándose este signo en siete de ellos. Los autores recomendaron corroborar la sospecha de neumotórax anterior con una proyección de pie en todos los pacientes en que se pesquisará este signo, que fue atribuido a la distribución subpulmonar del aire, más frecuente en los pacientes en posición supina ${ }^{(2)}$.

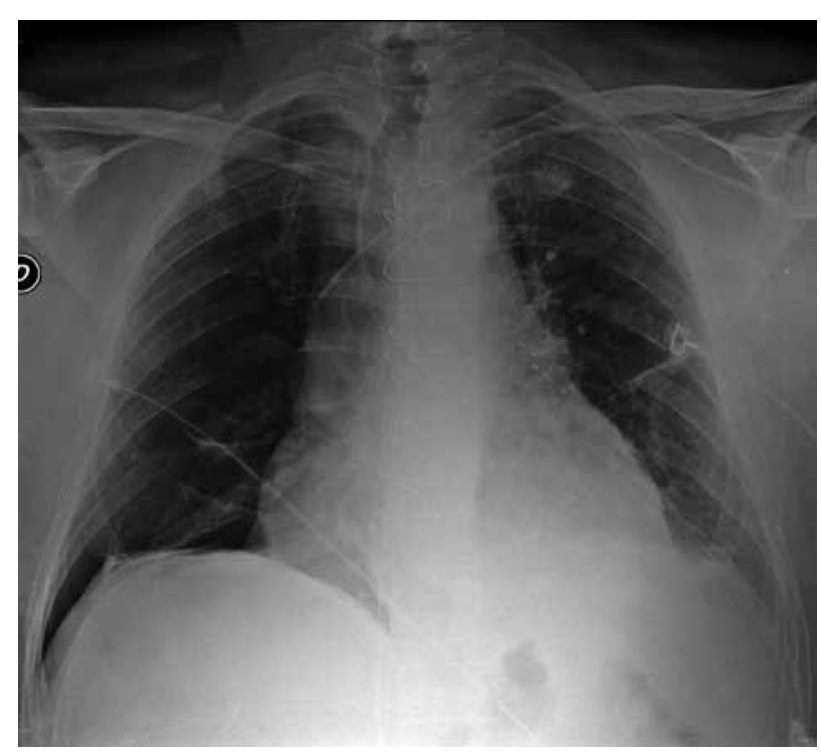

Figura 1. Rx de tórax AP decúbito supino de paciente adulto postoperado reciente de cirugía de revascularización miocárdica. Se observa distensión del receso costofrénico lateral derecho, que corresponde a signo del surco profundo. 


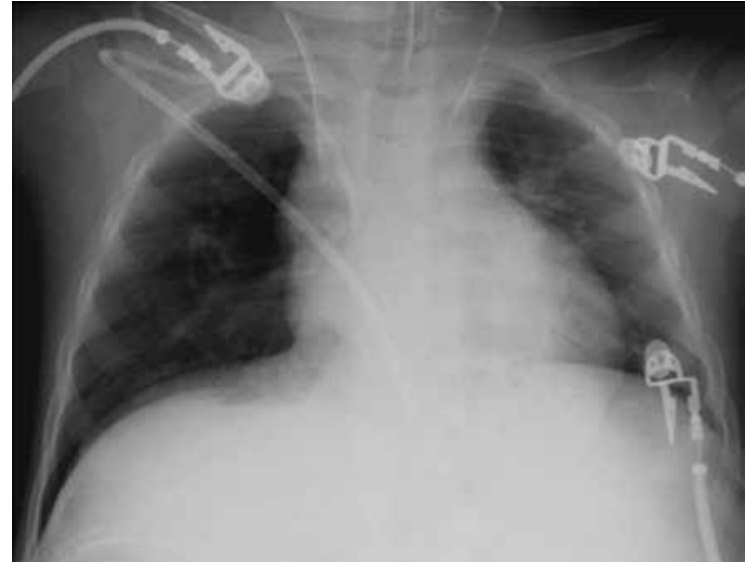

Figura 2. Rx de tórax obtenida en postoperatorio reciente de trasplante hepático en paciente pediátrico, evidencia signo del surco profundo, originado por neumotórax derecho.

\section{Significado}

Este signo traduce la presencia de aire en el espacio pleural (neumotórax), que se dispone en la porción no dependiente de la cavidad pleural en $\mathrm{Rx}$ de tórax efectuadas en decúbito dorsal (Figura 3). Como es sabido, con el paciente en bipedestación el neumotórax ocupará la región apicaldel hemitórax y en decúbito se dispondrá hacia anterior, a veces en situación medial adyacente al mediastino, subpulmonar y/o hacia lateral,pudiendo distender el seno costofrénico lateral en dirección caudal ${ }^{(3)}$ (Figura 4).
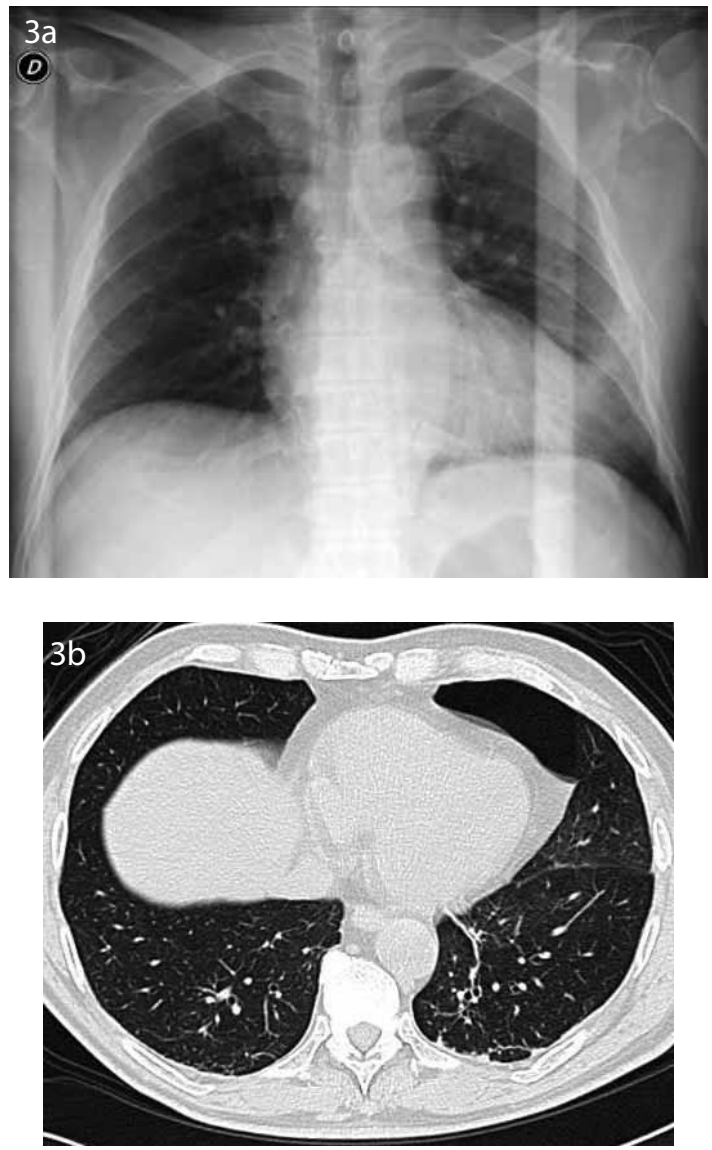

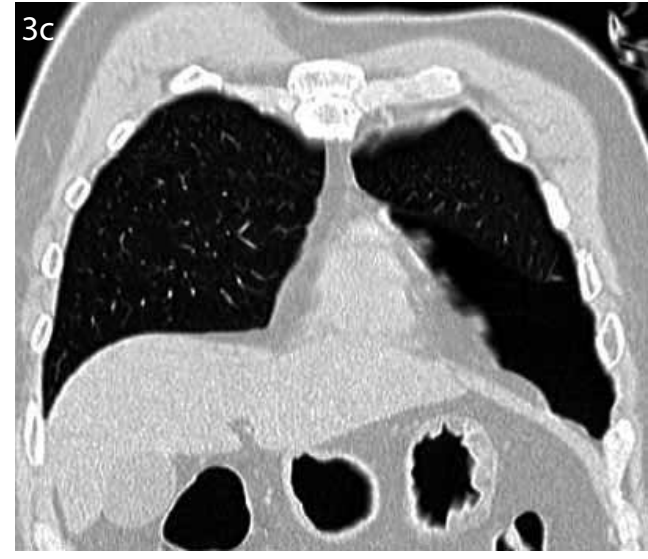

Figura 3. Paciente adulto con antecedente de accidente de tránsito. Rx de tórax portátil demuestra signo del surco profundo izquierdo, sugerente de neumotórax anterior (a), hallazgo que se corrobora en reconstrucciones axial (b) y coronal (c) de TC de tórax efectuada posteriormente.

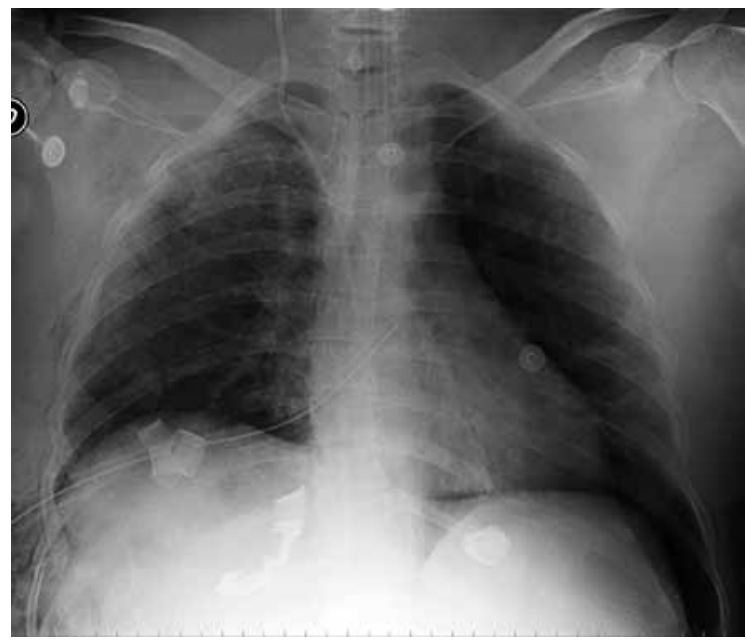

Figura 4. Rx de tórax AP decúbito de paciente adulto, politraumatizado. Presenta múltiples fracturas costales derechas y marcado aumento de la profundidad del receso costofrénico lateral izquierdo, debido a neumotórax de ese lado.

\section{Diagnóstico diferencial}

Los únicos falsos positivos descritos se refieren a pacientes adultos con hiperinsuflación secundaria a enfermedad pulmonar obstructiva crónica, en los que el atrapamiento aéreo distiende anormalmente los recesos costofrénicos laterales, ocasionalmente de forma asimétrica ${ }^{(2)}$.

\section{Discusión}

El neumotórax es una entidad muy frecuente en el contexto hospitalario, tanto en adultos como en niños. Dado que la gran mayoría de los pacientes críticos se controla con Rx de tórax portátiles toma- 
das en posición supina, se cree que hasta un $30 \%$ de los neumotórax de disposición anterior no son diagnosticados ${ }^{(3)}$. Para aumentar la pesquisa, resulta fundamental que las Rx portátiles de los pacientes críticos siempre incluyan toda la extensión de los ángulos costofrénicos y la región tóraco-abdominal.

La alta prevalencia de neumotórax en pacientes críticos, asociada a semiología sutil, hace fundamental un alto índice de sospecha diagnóstica, por lo que el radiólogo debe conocer los signos radiológicos y su utilidad. El diagnóstico de neumotórax se basa en la identificación, tanto en Rx como en tomografía computada (TC), de una fina opacidad lineal periférica que representa a la pleura visceral ("línea pleural") que corresponde al límite entre las hipodensidades dadas por el aire en el espacio pleural y el pulmón, con grados variables de atelectasia de acuerdo a la cuantía del neumotórax ${ }^{(4)}$. Este signo, uno de los más clásicos de la semiología radiológica torácica, resulta muy útil para pesquisar neumotórax en pacientes en bipedestación, pero se observa sólo en forma ocasional en Rx obtenidas en decúbito, en especial cuando el neumotórax es de gran cuantía ${ }^{(1)}$.

Otros signos descritos incluyen el "hemitórax radiolúcido", manifestación de una asimetría de transparencia que se origina en la acumulación de aire en toda la superficie anterior del espacio pleural ipsilateral y que puede ser difícil de observar en placas obtenidas en inspiración ${ }^{(4)}$. El aumento de la definición de los contornos mediastínico y/o diafragmático, por otro lado, ocurren cuando la distribución del aire en el espacio pleural es predominantemente medial y subpulmonar anterior, respectivamente ${ }^{(5)}$. Otros signos descritos son la banda radiolúcida paramediastínica y aquellos asociados al efecto de masa del espacio pleural ocupado ("pseudo-masas") sobre el parénquima pulmonar y el timo, en niños ${ }^{(6,7)}$.

En los recién nacidos, el neumotórax es frecuentemente secundario a patología pulmonar y/o uso de ventilación mecánica; en ellos, la escasa distensibilidad pulmonar dificulta el colapso compresivo del parénquima en casos de neumotórax y contribuye al aumento de volumen del hemitórax, por lo que en este grupo es más frecuente observar sobredistensión que puede asociarse a inversión diafragmática y a desplazamiento mediastínico ${ }^{(8)}$. En neonatos ha sido descrita también la herniación de la pleura parietal en la línea media anterior y la visualización de la línea de unión pleural anterior, en casos de neumotórax bilateral.

Cuando el signo del surco profundo está presente resulta de gran ayuda en estos pacientes, por lo que debe ser conocido por todos los radiólogos.

Comentarios

El diagnóstico radiológico de neumotórax dependerá de la posición en que fue obtenida la Rx y será mucho más complejo cuando ésta ha sido tomada en decúbito, especialmente cuando la cuantía del aire en el espacio pleural es menor.

El radiólogo deberá mantener una alta sospecha diagnóstica y conocer la semiología radiológica del diagnóstico de neumotórax, entre los cuales el signo del surco profundo resulta de gran utilidad en exámenes obtenidos en decúbito, tanto en adultos como en la población pediátrica.

Bibliografía

1. Kong A. The Deep Sulcus Sign. Radiology 2003; 228: 415-416.

2. Gordon R. The deep sulcus sign. Radiology 1980; 136: 25-27.

3. Sabbar S, Nilles E. Images in clinical medicine: Deep Sulcus Sign. N Engl J Med, 2012; 366(6): 552.

4. Moënne K, Ortega $X$. Diagnóstico por imágenes del tórax pediátrico. 2da Edición, 2012; 76-78.

5. Grim $\mathrm{P}$, Keenan W. Two uncommon radiographic signs of an anterior neonatal pneumothorax. Correlated with clinical finding. ClinPediatr 1986; 25: 440-444.

6. Webster R. Jr, Tonkin I. Lingular collapse with medial pneumothorax: puzzling image in children. AJR Am J Roentgenol 1981; 136: 919-921.

7. O'Keefe F, Swischuk L, Stransberry S. MediastinalPseudomass caused by compression of the thymus in neonates with anterior pneumothorax. AJR Am J Roentgenol 1990; 156: 145-149.

8. Fletcher B. Medial herniation of the parietal pleura: a useful sign of pneumothorax in supine neonates. AJR Am J Roentgenol 1978; 130: 469-472. 